\title{
INFLUÊNCIA DE CONDICIONANTES AMBIENTAIS NA SOBREVIVÊNCIA E INGRESSO DE INDIVÍDUOS DE Drosera brevifolia PURSH REALOCADOS EM FRAGMENTO DE CAMPO NATURAL
}

\author{
INFLUENCE OF ENVIRONMENTAL FACTORS ON THE SURVIVAL AND INFLOWS OF \\ INDIVIDUALS OF Drosera brevifolia PURSH REALLOCATED IN CAMPO NATURAL \\ FRAGMENT
}

\author{
Allan Rodrigo Nunho dos Reis ${ }^{1}$, Christopher Thomas Blum², Jaçanan Eloisa de Freitas Milani ${ }^{3}$ \\ 1,2,3 Universidade Federal do Paraná, Curitiba, Paraná, Brasil-allannunho@yahoo.com.br, \\ ctblum.ufpr@gmail.com \& jacanan.milani@gmail.com
}

\begin{abstract}
RESUMO
Drosera brevifolia Pursh é uma herbácea carnívora considerada ameaçada de extinção no município de Curitiba, Paraná. O objetivo do trabalho foi avaliar a influência das variáveis ambientais declividade, umidade do solo, temperatura e precipitação na sobrevivência de indivíduos de $D$. brevifolia realocados em Curitiba. Foi realizado o monitoramento mensal da sobrevivência pós-realocação e dos ingressos por regeneração natural ao longo de 12 meses. Foram implantados quatro tratamentos ( $T$ ) com 50 indivíduos cada, diferenciados pelo relevo, orientação e insolação: T1 (íngreme / sudoeste / pleno sol); T2 (íngreme / leste / sombra parcial); T3 (suave ondulado / leste / sombra parcial) e T4 (suave ondulado / sudoeste / pleno sol). Foram amostrados os solos e coletados dados de declividade e umidade do solo. 178 (89\%) das plantas morreram no primeiro mês após o plantio, devido às condições meteorológicas, com excesso de chuva nos primeiros dias (62 mm), seguido por ressecamento do solo causado pela forte insolação, com temperatura máxima de até $33,9^{\circ} \mathrm{C}$. A faixa de umidade do solo mais favorável ao estabelecimento de indivíduos realocados variou de $22 \%$ a $30 \%$. Apenas para o T1 foi verificada correlação positiva significativa entre a sobrevivência de plantas e as temperaturas, indicando que esta variável pode ser importante para o seu estabelecimento. Em nenhum tratamento foi constatada correlação significativa entre a sobrevivência e as variáveis precipitação, declividade e umidade do solo. Pode-se afirmar que $D$. brevifolia é sensível a pequenas mudanças nas condições ambientais locais.
\end{abstract}

PALAVRAS-CHAVE: Espécie ameaçada, planta carnívora, transplantio

\section{ABSTRACT}

Drosera brevifolia Pursh is a carnivorous herbaceous plant considered to be endangered in the municipality of Curitiba, Parana. The aim of this work was to evaluate the influence of environmental variables slope, soil moisture, temperature and precipitation on the survival of individuals of $D$. brevifolia reallocated in Curitiba. Monthly monitoring of post-relocation survival and natural regeneration inflows over 12 months was performed. Four treatments $(\mathrm{T})$ were implanted with each of the 50 individuals, differentiated by relief, orientation and sunshine: T1 (steep / southwest / full sun); T2 (steep / east / partial shade); T3 (smooth wavy / east / partial shade) and T4 (smooth wavy / southwest / full sun). Soils and data groups of slope and soil moisture were sampled. 178 (89\%) of the plants died on the first day after planting due to meteorological conditions, with an increase of rainfall in the first days $(62 \mathrm{~mm})$, followed by dryness of the soil by the strong insolation, with maximum temperature up to 33.9 ${ }^{\circ} \mathrm{C}$. The soil moisture range more favorable to the establishment of reallocated individuals ranged from $22 \%$ to $30 \%$. Only for T1 was a significant positive correlation between plant survival and temperature, indicating that this variable may be important for its establishment. No significant correlation was observed between survival and rainfall, slope and soil moisture variables. It can be stated that $D$. brevifolia is sensitive to small changes in local environmental conditions.

KEYWORDS: Carnivorous plant, threatened species, transplanting. 


\section{INTRODUÇÃO}

A compreensão da relação biológica existente entre uma espécie rara ou ameaçada de extinção com o ambiente onde se encontra e da situação atual de sua população são fundamentais para a sua proteção, possibilitando a identificação dos fatores que a colocam em risco (PRIMACK \& RODRIGUES, 2001).

Dentre os grupos de plantas que mais sofrem com ameaças de extinção está o das plantas carnívoras, devido a sua grande exigência quanto à qualidade ambiental (FERREIRA et al., 2013). Como agravante, tem-se que aproximadamente $80 \%$ das espécies de plantas carnívoras conhecidas, cerca de 750, ainda não compõem a Lista Vermelha de Espécies Ameaçadas de Extinção da International Union for Conservation of Nature (IUCN), o que dificulta a elaboração de estratégias de conservação (IUCN, 2015).

Uma das famílias pertencentes a este grupo é Droseraceae, representada no Brasil apenas pelo gênero Drosera L. (SILVA \& GIULIETTI, 1997). Suas espécies apresentam glândulas, presentes principalmente na face adaxial das folhas, cobertas por gotas de uma secreção mucilaginosa ácida e enzimática, responsáveis pela captura de insetos (CROWDER et al., 1990; SILVA \& GIULIETTI, 1997).

O gênero Drosera L. possui aproximadamente 220 espécies descritas, com presença em todos os continentes (JUNIPER et al., 1989; ROYAL BOTANIC GARDEN, 2016). No Brasil, ocorrem 31 espécies desta divisão, sendo que 19 são endêmicas (SILVA, 2016). Segundo Kaehler et al. (2014), sete espécies do gênero já foram descritas no estado do Paraná.

Algumas espécies de Drosera L. já foram enquadradas sob risco de extinção devido a diversas razões, como as atividades ligadas à agricultura, à pecuária, à produção de energia e à mineração; à urbanização; à poluição; às modificações nos sistemas naturais; à competição com espécies invasoras e ao uso indiscriminado em indústrias de chás, em países do sudeste asiático (JENNINGS \& ROHR, 2011; MAJUMDAR et al., 2011).

Existe uma estreita relação entre a ocorrência das espécies deste gênero com solos ácidos, pobres em nutrientes e com suprimento hídrico contínuo, pelo menos durante o período de crescimento das plantas (ADAMEC, 1997; SARIDAKIS et al., 2004).

Saridakis et al. (2014) acrescentam que, embora a nutrição de Drosera dependa parcialmente da captura de insetos, estas plantas também interagem sinergicamente com grupos de microrganismos associados à rizosfera, o que pode contribuir para o suprimento de nutrientes em ambientes oligotróficos, auxiliando a planta a se manter em solos de baixa fertilidade.

Drosera brevifolia Pursh possui hábito herbáceo terrícola, sendo classificada como heliófila e seletiva higrófita (SANTOS, 1980). Apresenta distribuição disjunta e ocorre no sul dos Estados Unidos, México, Belize, Cuba, Uruguai e na região sul do Brasil, em campos arenosos de regiões litorâneas ou em campos úmidos de planalto (SILVA \& GIULIETTI, 1997; GONELLA, 2012). Os indivíduos de $D$. brevifolia se estabelecem comumente em locais restritos e distintos ou com pequenas variações entre os micro-habitats (SARIDAKIS et al., 2004).

A dispersão de $D$. brevifolia ocorre de forma epizoocórica (JUNIPER et al., 1989). A espécie diferenciase das demais espécies do gênero principalmente por geralmente não apresentar estípulas, sendo estas reduzidas, quando presentes (ARROYO \& SILVA, 2005).

$D$. brevifolia pode encontrar-se localmente ameaçada de extinção na região de Curitiba, devido aos poucos registros de tombamento encontrados em herbários e ao tempo decorrido desde a última coleta realizada neste município, há cerca de 20 anos (CENTRO DE REFERÊNCIA EM INFORMAÇÃO AMBIENTAL (CRIA), 2016). Tal constatação pode ser explicada pela pouca quantidade de remanescentes de vegetação de campo natural, que originalmente cobriam boa parte da área de Curitiba e que, há décadas vem sofrendo pressões antrópicas (KLEIN \& HATSCHBACH, 1962).

De fato, considera-se que a maior ameaça às populações desta espécie na região sejam a destruição do seu habitat pelas atividades humanas (SARIDAKIS et al., 2004). Assim, Stefano \& Silva (2001), recomendam que a espécie $D$. brevifolia seja considerada ameaçada de extinção onde ocorre, devido a estes locais, em geral sofrerem fortes impactos antrópicos ou serem ambientalmente vulneráveis.

Tendo em vista este contexto, o presente trabalho teve como objetivo verificar se existe influência das variáveis ambientais declividade, umidade do solo, temperatura e precipitação na sobrevivência de indivíduos de $D$. brevifolia realocados de uma área alterada em Curitiba, no Estado do Paraná.

\section{MATERIAL E MÉTODOS}

\section{Caracterização da área}

O estudo foi realizado em um remanescente de vegetação natural herbáceo-arbustiva no Campus III da 
Universidade Federal do Paraná (UFPR), em Curitiba, Paraná, onde há a presença de pequenos fragmentos de vegetação de campo natural.

A cidade de Curitiba está inserida a aproximadamente $25^{\circ} 25^{\prime} 40^{\prime \prime}$ de latitude sul e $49^{\circ} 16^{\prime} 23^{\prime \prime}$ de longitude oeste, dentro do Primeiro Planalto Paranaense, na área de abrangência do bioma Mata Atlântica (INSTITUTO DE PESQUISA E PLANEJAMENTO URBANO DE CURITIBA (IPPUC), 2011). Conforme a classificação de KöppenGeiger, o clima regional é do tipo $\mathrm{Cfb}$, subtropical úmido, mesotérmico, sem estação seca, verões frescos e invernos com geadas frequentes e precipitações de neve ocasionais (IPPUC, 2011). As temperaturas médias são de $19,7{ }^{\circ} \mathrm{C}$ no verão e $13,4{ }^{\circ} \mathrm{C}$ no inverno, enquanto a precipitação média anual é de 1.419,91 mm (IPPUC, 2011; ZAMPRONI et al., 2013).

Grande parte da porção leste de Curitiba, onde se localiza a área de estudo, era originalmente coberta por campos naturais, com vegetação constituída por espécies de gramíneas sobre relevo suave-ondulado, entremeados por agrupamentos de espécies arbóreas às margens dos rios ou isolados sobre o campo, com formas e dimensões variadas e predomínio de Araucaria angustifolia (Bertol.) Kuntze (KLEIN \& HATSCHBACH, 1962; RODERJAN et al., 2002; RONDON NETO et al., 2002; SELUSNIAKI \& ACRA, 2010). Os solos do local de estudo foram descritos por Rondon Neto et al. (2002), que verificaram a predominância de Cambissolos.

\section{Procedimentos metodológicos}

O experimento consistiu na avaliação da sobrevivência e ingresso por regeneração natural de indivíduos de $D$. brevifolia (Figura 1) transplantados a partir de um terreno sob processo de supressão da vegetação, com presença de solos da classe dos Cambissolos, próximo à área de estudo, em setembro de 2014. Após seu resgate, 200 indivíduos (cinco repetições de 10 indivíduos por tratamento) foram plantados em áreas do Campus III que combinavam distintas situações de relevo, exposição e insolação: T1 (íngreme / sudoeste / pleno sol); T2 (íngreme/ leste/ sombra parcial); T3 (suave ondulado / leste / sombra parcial); e T4 (suave ondulado / sudoeste / pleno sol). As dimensões dos tratamentos foram $100 \times 50$ $\mathrm{cm}$, sendo os indivíduos transplantados sistematicamente a cada $10 \mathrm{~cm}$ em relação às bordas. Após o plantio, foi realizado monitoramento mensal de sobrevivência e ingresso de novos indivíduos durante 12 meses por meio da contagem de plantas dentro das parcelas.

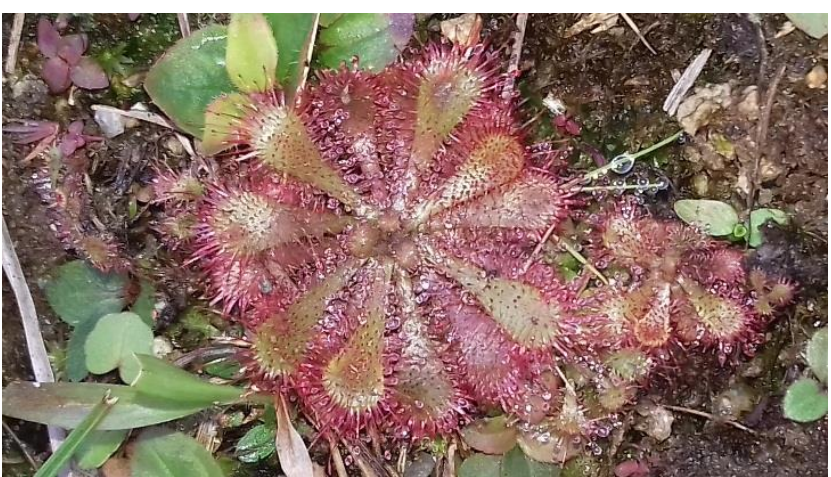

Figura 1. Indivíduos de Drosera brevifolia após 9 meses da implantação do experimento, Curitiba, Paraná.

Foi utilizado um trado holandês para as coletas de solos, com posterior classificação segundo o Sistema Brasileiro de Classificação de Solos (INSTITUTO BRASILEIRO DE GEOGRAFIA E ESTATíSTICA (IBGE), 2015). $O$ percentual de umidade no solo foi obtido por meio da pesagem de amostras de peso úmido e seco. As amostras foram coletadas em maio de 2016, após um período de chuvas. Foram retiradas cinco amostras de solo com $5 \mathrm{~cm}$ de profundidade de cada tratamento. A profundidade de coleta foi definida a partir da observação do sistema radicular da espécie. As amostras de solo foram acondicionadas em recipientes de alumínio e pesadas em balança digital com precisão de 0,01 g antes e após a secagem em estufa a $105{ }^{\circ} \mathrm{C}$ por 24 horas (BROWER \& ZAR, 1977).

A declividade dos taludes foi obtida pelo método trigonométrico, medindo-se as distâncias horizontal e vertical das extremidades dos taludes, formando um ângulo de $90^{\circ}$. A inclinação foi obtida dividindo-se a distância vertical pela distância horizontal, e em seguida calculou-se a função tangente em seu inverso, o arcotangente, obtendo-se o valor do ângulo em graus. Estes valores foram enquadrados em classes de declividade segundo IBGE (2015).

As variáveis meteorológicas utilizadas foram os valores mensais de mínimas, médias e máximas de temperatura e acumulados de precipitação (Figura 2), obtidos na estação meteorológica do Instituto Tecnológico SIMEPAR (Sistema Meteorológico do Paraná), situada a menos de $1.000 \mathrm{~m}$ da área de estudo.

Foi testada a relação entre as variáveis ambientais e a sobrevivência por meio da correlação de Spearman, ao final dos 12 meses de monitoramento, sendo que para o teste da correlação entre a sobrevivência, a precipitação e a temperatura, não foram utilizados os dados do Tratamento 3, pois não haviam mais indivíduos vivos pouco após o início do experimento. 


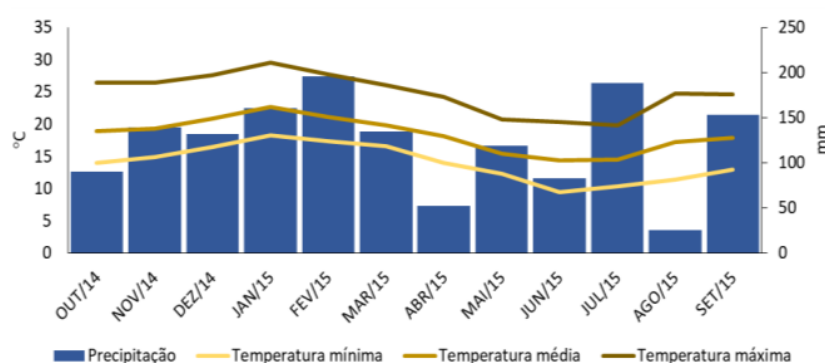

Figura 2. Dados meteorológicos mensais do período de estudo: outubro de 2014 a setembro de 2015.

Fonte: Simepar.

Realizou-se uma análise considerando a sobrevivência em função do local e dos dias após o plantio (DAP). O experimento consistiu-se de um ensaio unifatorial em delineamento inteiramente casualizado, conduzido em cinco repetições. $O$ fator de estudo corresponde ao local de plantio e a variável aleatória analisada foi a sobrevivência das plantas aos 701 dias após o plantio. Essa, por sua vez, não é homogênea em termos de sua variância, não sendo possível se aproximar de variâncias homogêneas por meio da transformação dos dados, uma vez que as repetições ao longo de um tratamento não diferem entre si em termos de sua observação.

Por esse motivo optou-se pela aplicação do teste nãoparamétrico de Kruskal-Wallis (BENAYAS et al., 2009), nível de significância de $95 \%$. Os dados de ingresso dos indivíduos da espécie foram avaliados por meio de uma regressão logística, considerando como variável dependente o ingresso e como fatores as declividades letra a. (íngreme / sudoeste) e letra b. (íngreme / leste). Para as análises estatísticas utilizou-se o programa STATGRAPHICS Centurion XVI.I (STATPOINT TECHNOLOGIES, 2010).

\section{RESULTADOS E DISCUSSÃO}

Quanto à amostragem dos solos, identificou-se que estes compõem a classe dos Cambissolos, caracterizados por apresentaram um horizonte $B$ incipiente $(\mathrm{Bi})$ e serem, em geral, rasos e com drenagem lenta (IBGE, 2015). Os resultados encontrados nas análises de declividade e umidade do solo estão apresentados na Tabela 1.

Em relação à umidade do solo, verificou-se que os maiores teores têm relação direta com o gradiente de declividade do terreno, sendo que as partes mais baixas deste possuem umidades superiores. Isto ficou claro ao se analisar de forma separada o T1, onde a média das três amostras retiradas na porção superior resultou em 19,5\%, enquanto que na porção inferior do tratamento, a média das três amostras foi de 33,1\%.

Tabela 1. Características ambientais dos tratamentos dos experimentos de sobrevivência pós-relocação de D. brevifolia

\begin{tabular}{cccc}
\hline Tratamento & Descrição & Declividade & $\begin{array}{c}\text { Umidade } \\
\text { do solo (\%) }\end{array}$ \\
\hline T1 & $\begin{array}{c}\text { Plantio de indivíduos } \\
\text { isolados; encosta } \\
\text { fortemente montanhosa } \\
\text { Plantio de indivíduos } \\
\text { isolados; encosta } \\
\text { T2 }\end{array}$ & $55^{\circ}$ & 23,88 \\
& $\begin{array}{c}\text { fortemente montanhosa } \\
\text { Plantio de indivíduos } \\
\text { T3olados; terreno } \\
\text { suave ondulado }\end{array}$ & $5^{\circ}$ & 22,27 \\
& $\begin{array}{l}\text { Plantio de indivíduos } \\
\text { isolados; terreno } \\
\text { suave ondulado }\end{array}$ & 55,50 \\
\hline
\end{tabular}

Pôde-se constatar uma drástica diminuição na quantidade de indivíduos de $D$. brevifolia desde seu plantio. Logo nas primeiras semanas muitas plantas morreram, cerca de 178 (89\%), possivelmente devido ao estresse causado pela realocação, mas também devido às condições meteorológicas extremas daquele período, com excesso de chuva nos primeiros dias $(62 \mathrm{~mm})$, o que afetou os tratamentos em áreas de taludes suaves (T3 e T4) e ressecamento do solo causado pela forte insolação, com temperatura máxima absoluta chegando a $33,9^{\circ} \mathrm{C}$, o que dizimou indivíduos plantados nos taludes íngremes (T1 e T2), como pode ser visualizado na Figura 3. Tal evento indica que a espécie é muito sensível a flutuações ambientais pontuais. Verificou-se ainda que, a princípio, a exposição não influenciou a sobrevivência das plantas.

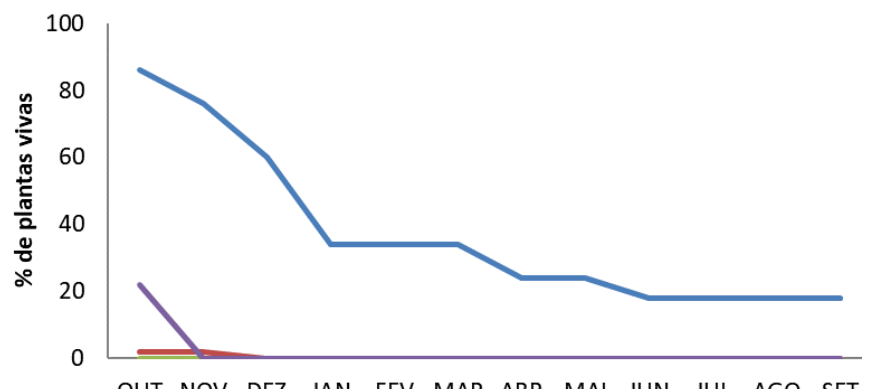$$
-\mathrm{T} 1-\mathrm{T} 2-\mathrm{T} 3-\mathrm{T} 4
$$

Figura 3. Sobrevivência dos indivíduos de $D$. brevifolia, no primeiro ano após o plantio de relocação, entre outubro de 2014 a setembro de 2015.

Este resultado corrobora o encontrado no estudo de Godwin (2011), com a espécie Drosera capillaris Poir., onde foi verificado que a disponibilidade de água é um fator limitante ao estabelecimento da espécie. Tal 
constatação também condiz com o trabalho de Ridder \& Dhondt (1992), o qual indicou que a dinâmica demográfica de populações de Drosera intermedia Hayne pode ser explicada por diferenças na sobrevivência de adultos e no ingresso por reprodução sexuada, relacionando-se a fatores bióticos e abióticos específicos do local, como as secas e períodos de alta intensidade de precipitação. Schnell (1976) acrescenta que é comum os indivíduos adultos de $D$. brevifolia morrerem durante o verão se houverem períodos prolongados de seca, sendo que as sementes germinam no ano seguinte.

Não foram constatadas correlações significativas entre os valores mensais de sobrevivência e as variáveis precipitação e temperaturas máxima, média e mínima. A única exceção foi para o T1, cuja sobrevivência de plantas teve correlação positiva significativa com as temperaturas máxima, média e mínima ( $p=0,0128,0,0166$ e 0,0236, respectivamente). É provável que somente este tratamento tenha apresentado correlação significativa pelo fato de possuir maior número de indivíduos vivos, uma vez que nos demais tratamentos a sobrevivência foi muito baixa. Tal constatação pode também ter relação com a escala dos dados meteorológicos utilizada, que pode não ter sido capaz de indicar as variações encontradas nos microhabitats, conforme verificado por Saridakis et al. (2004).

Também não foi demonstrada correlação significativa entre as variáveis declividade e umidade do solo com a sobrevivência ao final de 12 meses em todos os tratamentos. No entanto, mesmo sem a constatação de correlações significativas, pode-se inferir que dentre os teores de umidade do solo constatados neste experimento, o mais favorável para a sobrevivência de indivíduos realocados é de cerca de $24 \%$ (T1), o que também permite considerar que ambientes de menor declividade e com possibilidade de saturação excessiva de água no solo (T3 e T4), com valores superiores a $35 \%$, podem ser desfavoráveis à sobrevivência de $D$. brevifolia.

Em estudo realizado por Wolf et al. (2006), verificouse que a espécie Drosera rotundifolia L. sobrevive exclusivamente em zonas obrigatoriamente úmidas, com entradas subterrâneas de água estáveis e com níveis freáticos quase superficiais, devido as suas raízes não tolerarem a dessecação. Outra espécie do gênero, Drosera montana A.St.-Hil., apresenta maior abundância de indivíduos em condições de umidade intermediária (de $20 \%$ a $25 \%$ ) do solo, o que reflete sua limitação por disponibilidade de água (ESPÍRITO-SANTO \& WERNECK, 1999). Estes resultados corroboram os encontrados no presente estudo, onde se observou maior sucesso no estabelecimento dos indivíduos realocados para os locais de umidade mediana no solo.

Somente foi registrado ingresso de plantas nos tratamentos T1 e T2 (Figura 4), porém os indivíduos ingressantes no $\mathrm{T} 2$ não resistiram e morreram ao final do acompanhamento. Nos demais, além da mortalidade de todos os indivíduos plantados, não houve ingressos, possivelmente devido à grande mortalidade inicial dos indivíduos e ao curto espaço de tempo para a formação de banco de sementes, pois, segundo Ridder \& Dhondt (1992), as espécies do gênero Drosera recolonizam rapidamente os locais após alterações na superfície do solo, embora apresentem baixa capacidade de dispersão das sementes, sendo o ingresso de indivíduos o resultado de um banco de sementes persistente.

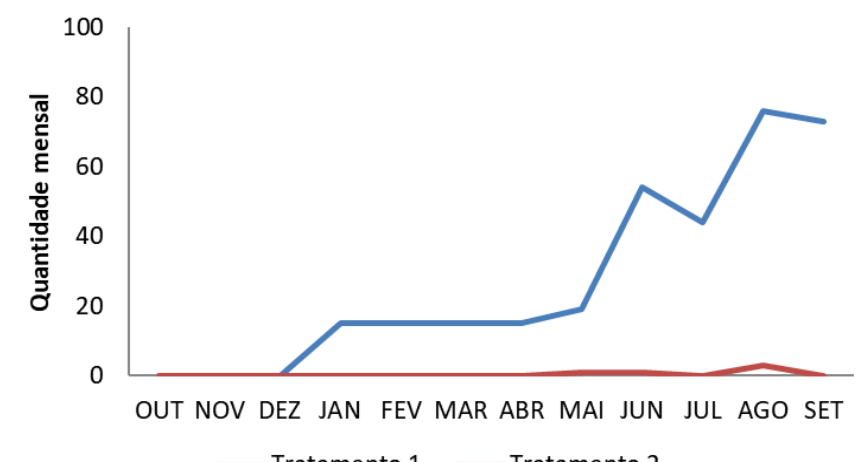

Figura 4. Quantidade mensal de indivíduos de D. brevifolia ingressantes nos tratamentos T1 (a) e T2 (b), entre outubro de 2014 e setembro de 2015.

Foi verificado por Ridder \& Dhondt (1992) que a sobrevivência de indivíduos de $D$. intermedia é influenciada principalmente pela idade, pelo modo reprodutivo (clonal ou por sementes) e pelas interações entre esses fatores e ainda que os ingressos bemsucedidos são muito variáveis de ano para ano, não sendo detectados em períodos curtos, como o avaliado no presente estudo.

Quando considerada a sobrevivência em função do local e dos dias após o plantio (DAP), verificou-se uma taxa de sobrevivência de $20,9 \%$ das plantas alocadas no talude com declividade $55^{\circ}$ e orientação sudoeste (T1). Tal percentual de sobrevivência, ainda que pequeno, deve-se ao fato de os locais de micro-relevo acidentado não representarem uma limitação ao estabelecimento das plantas carnívoras.

Como observado por Zamora (2002) estas plantas comumente habitam terrenos declivosos, inclusive paredes rochosas, sendo que estes locais condicionam a ecologia das populações situadas sob diferentes graus de 
exposição solar, fato reforçado pelo estudo realizado por Fernández et al. (2008), onde se verificou que declividades elevadas também são locais propícios para o estabelecimento da espécie carnívora Pinguicula vallisneriifolia Webb, nesta mesma orientação (sudoeste).

$\mathrm{O}$ teste de Kruskal-Wallis indicou diferença estatística na sobrevivência das plantas nas distintas áreas em estudo (Tabela 2), indicando que o T1 é superior aos demais tratamentos em relação às condições ambientais para o estabelecimento da espécie.

Tabela 2. Diferenciação das médias de sobrevivência entre os tratamentos

\begin{tabular}{ccc}
\hline Tratamento & Média dos postos & $\begin{array}{c}\text { Médias de } \\
\text { sobrevivência (\%) }\end{array}$ \\
\hline T1 & $18 \mathrm{a}$ & 20,90 \\
T2 & $8 \mathrm{~b}$ & 0,00 \\
T3 & $8 \mathrm{~b}$ & 0,00 \\
T4 & $8 \mathrm{~b}$ & 0,00 \\
\hline
\end{tabular}

Valor da estatística de Kruskal-Wallis 19,00**.

Os resultados da regressão logística indicaram que ocorreu um maior ingresso de indivíduos da espécie no tratamento T1 do que no tratamento T2 (Figura 5).

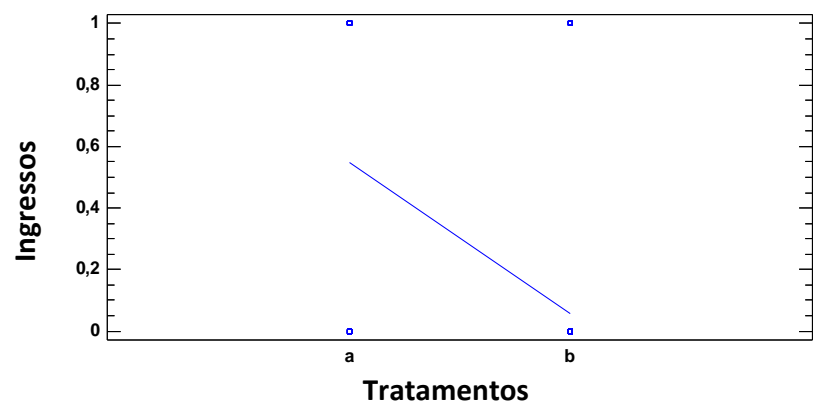

Figura 5. Regressão logística para os dados de ingresso de D. brevifolia. (a) T1 (íngreme / sudoeste / pleno sol) e (b) T2 (íngreme / leste / sombra parcial).

Foi possível inferir que $26 \%$ do ingresso de indivíduos é regulado pela declividade, confirmado pela razão de verossimilhança de 0,0000 , pertencentes ao tratamento. Como o valor de $p<0,05$, esse termo é estatisticamente significativo ao nível de confiança de $95 \%$.

\section{CONCLUSÕES}

A grande mortalidade inicial dos indivíduos realocados demonstra que $D$. brevifolia é extremamente sensível a pequenas mudanças nas condições ambientais locais.

A correlação significativa com temperatura no tratamento com maior sobrevivência indica que esta variável possa ser importante para o estabelecimento de populações desta espécie.

O teor de umidade em torno de $24 \%$, valor mediano, aparenta ser propício para a sobrevivência de indivíduos transplantados de $D$. brevifolia.

Plantios em taludes íngremes não representam um problema para a espécie, uma vez que foi nestes ambientes onde ocorreu a maior sobrevivência de indivíduos realocados e a maior quantidade de ingressantes. Por outro lado, locais com possibilidade de encharcamento do solo são desfavoráveis à espécie.

\section{REFERÊNCIAS}

ADAMEC, L. Mineral nutrition of carnivorous plants: a review. The Botanical Review, v.63, n.3, p.273-299, 1997.

ARROYO, M.D.C.; SILVA, T.R.S. Drosera (Droseraceae). New York: Flora Neotropica, 2005.

BENAYAS, J.M.R. et al. Enhancement of biodiversity and ecosystem services by ecological restoration: a meta-analysis. Science, v.325, n. 1121, 2009.

BROWER, J.E.; ZAR, J.H. Field and laboratory methods for general ecology. lowa: W. C. Brown Company Publishers, 1977.

CRIA - Centro de Referência em Informação Ambiental. SpeciesLink: Drosera brevifolia, 2016. Disponível em: http://www.splink.org.br/index?lang=pt

CROWDER, A.A. Drosera L. Journal of Ecology, v.78, p.233-267, 1990.

FERNÁNDEZ, J.B. et al. Pinguicula vallisneriifolia Webb (Lentibulariceae), nueva especie para la flora valenciana. Flora Montiberica, v.40, p.34-37, 2008.

FERREIRA, L.V. et al. Primeira ocorrência de Drosera cayennensis Sagot ex Diels (Droseraceae) nas campinas do baixo rio Tocantins, estado do Pará, como subsídio à criação de novas unidades de conservação. Boletim do Museu Paraense Emílio Goeldi Ciências Naturais, v.8, p.223-230, 2013.

GODWIN, R.L. Investment in carnivory in Drosera capillaris: effects of varying light levels on trichome density, 2011. 43p. (Dissertação de mestrado).

GONELLA, P.M. Revisão taxonômica do Clado TetraploideBrasileiro de Drosera L. (Droseraceae), 2012. 237p. (Dissertação de mestrado).

IBGE - Instituto Brasileiro de Geografia e Estatística. Manual Técnico de Pedologia. 3 ed. Rio de janeiro: IBGE, 2015.

IPPUC - Instituto de Pesquisa e Planejamento Urbano de Curitiba. Desenvolvimento sustentável: indicadores de sustentabilidade de Curitiba - 2010. Curitiba: IPPUC, 2011.

IUCN - International Union for Conservation of Nature. 
Carnivorous plants need help! 2015. Disponível em: http://support.iucnredlist.org/carnivorousplants

JENNINGS, D.E.; ROHR, J.R. A review of the conservation threats to carnivorous plants. Biological Conservation, v.144, p.13561363, 2011.

JUNIPER, B.E. et al. The Carnivorous Plants. London: Academic Press, 1989.

KAEHLER, M.; GOLDENBERG, R.; EVANGELISTA, P.H.L.; RIBAS, O.S.; VIEIRA, A.O.S.; HATSCHBACH, G.G. Plantas vasculares do Paraná. Curitiba: Departamento de Botânica da UFPR, 2014.

KLEIN, R.M.; HATSCHBACH, G. Fitofisionomia e notas sobre a vegetação para acompanhar a planta fitogeográfica do município de Curitiba e arredores (Paraná). Boletim da Universidade do Paraná. Geografia Física, n.4, 1962.

MAJUMDAR, K. et al. Community structure and population status of Drosera burmanii Vahl. with new distributional record in Tripura, Índia. Journal of Ecology and the Natural Environment, v.3, p.410-414, 2011.

PRIMACK, R.B.; RODRIGUES, E. Biologia da Conservação. Londrina: Editora Planta, 2001.

RIDDER, F.; DHONDT, A.A. The demography of a clonal herbaceous perennial plant, the longleaved sundew Drosera intermedia, in different heathland habitats. Ecography, v.15, p.129-143, 1992.

RODERJAN, C.V. et al. As unidades fitogeográficas do Estado do Paraná. Ciência \& Ambiente, v.24, p.75-92, 2002.

RONDON NETO, R.M. Caracterização florística e estrutural de um fragmento de floresta ombrófila mista, em Curitiba, PR - Brasil. Floresta, v.32, n.1, p.3-16, 2002.

ROYAL BOTANIC GARDEN. Plants of the world online: Drosera $\mathrm{L}$. 2016. Disponível em: http://powo.science.kew.org/taxon/urn: Isid:ipni.org:names:30001036-2

SANTOS, E. Droseráceas. In: REITZ R. Flora ilustrada catarinense. Herbário Barbosa Rodrigues. Itajaí: IOESC. p.18-22, 1980.

SARIDAKIS, D.P. et al. Microhabitat preferences of six Drosera (Droseraceae) from Tibagi River Basin, Paraná State, Brazil. Brazilian Archives of Biology and Technology, v.47, p.495-501, 2004.

SARIDAKIS, D.P. et al. An appraisal on saprophytic and functional microbial communities associated to the carnivorous plant Drosera latifolia (Eichler) Gonella \& Rivadavia (Droseraceae). Semina: Ciências Biológicas e da Saúde, v.35, n.1, p.3-14, 2014.

SCHNELL, D.E. Carnivorous plants of the United States and Canada. Winston-Salem: John F. Blair, 1976.

SELUSNIAKI, M.; ACRA, L.A. O componente arbóreo-arbustivo de um remanescente de Floresta com Araucária no município de Curitiba, Paraná. Floresta, v.40, n.3, p.593-602, 2010.

SILVA, T.R.S. Droseraceae. Rio de Janeiro: Jardim Botânico do
Rio de Janeiro, 2016. Disponível em: http://reflora.jbrjgov.br/ja bot/floradobrasil/FB7420

SILVA, T.R.S.; GIULIETTI, A.M. Levantamento das Droseraceae do Brasil. Boletim de Botânica da Universidade de São Paulo, v.16, p.75-105, 1997.

STATPOINT TECHNOLOGIES. Statgraphics Centurion XVI.I, 2010. Disponivel em: http://www.statgraphics.com

STEFANO, R.D.; SILVA, T.R.S. Conservation status of the carnivorous genus Drosera in South America and the Caribbean. Harvard Papers in Botany, v.6, n.1, p.253-260, 2001.

WOLF, E. et al. Drosera rotundifolia L. (roundleaf sundew): a technical conservation assessment. Rocky Mountain Region: USDA Forest Service, 2006. Disponível em: http://www.fs.usda.gov/Internet/FSE_DOCUMENTS/stelprdb52 50874.pdf

ZAMPRONI, K. et al. Efeito das variáveis meteorológicas sobre a fenologia de Tipuana tipu (Benth.) O. Kuntze na arborização urbana de Curitiba - PR. Revista Brasileira de Arborização Urbana, v.8, p.1-14, 2013. 